\title{
MCViNE - An object oriented Monte Carlo neutron ray tracing simulation package
}

\author{
Jiao Y. Y. Lin ${ }^{a, b, c}$, Hillary L. Smith ${ }^{\mathrm{b}}$, Garrett E. Granroth ${ }^{\mathrm{c}}$, \\ Douglas L. Abernathy ${ }^{\mathrm{d}}$, Mark D. Lumsden ${ }^{\mathrm{d}}$, Barry Winn ${ }^{\mathrm{d}}$, Adam A. Aczel ${ }^{\mathrm{d}}$, \\ Michael Aivazis ${ }^{\mathrm{a}}$, Brent Fultz ${ }^{\mathrm{b}}$ \\ ${ }^{a}$ Caltech Center for Advanced Computing Research, California Institute of Technology \\ ${ }^{b}$ Department of Applied Physics and Materials Science, California Institute of Technology \\ ${ }^{c}$ Neutron Data Analysis and Visualization Division, Oak Ridge National Laboratory \\ ${ }^{d}$ Quantum Condensed Matter Division, Oak Ridge National Laboratory
}

\begin{abstract}
MCViNE (Monte-Carlo VIrtual Neutron Experiment) is an open-source Monte Carlo (MC) neutron ray-tracing software for performing computer modeling and simulations that mirror real neutron scattering experiments. We exploited the close similarity between how instrument components are designed and operated and how such components can be modeled in software. For example we used object oriented programming concepts for representing neutron scatterers and detector systems, and recursive algorithms for implementing multiple scattering. Combining these features together in MCViNE allows one to handle sophisticated neutron scattering problems in modern instruments, including, for example, neutron detection by complex detector systems, and single and multiple scattering events in a variety of samples and sample environments. In addition, MCViNE can use simulation components from linear-chain-based MC ray tracing packages which facilitates porting instrument models from those codes. Furthermore it allows for components written solely in Python, which expedites prototyping of new components. These developments have enabled detailed simulations of neutron scattering experiments, with non-trivial samples, for time-of-flight inelastic instruments at the Spallation Neutron Source.
\end{abstract}

\footnotetext{
* Corresponding author

Email addresses: linjiao@ornl.gov, linjiao@caltech.edu (Jiao Y. Y. Lin ), granrothge@ornl.gov (Garrett E. Granroth), btf@caltech.edu (Brent Fultz)
}

Preprint submitted to NIMA

October 8, 2015

(C) 2015. This manuscript version is made available under the Elsevier user license http://www.elsevier.com/open-access/userlicense/1.0/ 
Examples of such simulations for powder and single-crystal samples with various scattering kernels, including kernels for phonon and magnon scattering, are presented. With simulations that closely reproduce experimental results, scattering mechanisms can be turned on and off to determine how they contribute to the measured scattering intensities, improving our understanding of the underlying physics.

Keywords: neutron scattering, Monte Carlo simulation, ray-tracing, inelastic, spectrometry

\section{Introduction}

Data analysis for neutron time-of-flight spectroscopy has predominantly been a conversion of raw data to intensities with instrument independent units; namely $S(\mathbf{Q}, \omega)$ or $S(\mathbf{Q}, E)$, where $S$ is the dynamical structure factor, and

${ }_{5} \mathbf{Q}, \omega, E$ are the wave vector, frequency, and energy. This approach has been the basis for many studies, but some cases require a more elaborate analysis scheme (see, for example, $[1,2,3,4,5,6,7,8,9]$ ).

More specifically, traditional reduction assumes single scattering, that the resolution function can be modeled by a simple function, that the scattering process of interest can be readily separated from other scattering processes in the system, and that scattering from the sample environment is negligible or easily subtracted. Monte Carlo ray-tracing can provide a straightforward way to perform analysis in cases where some, or all, of the above assumptions are not valid. Specifically it can be used to investigate complex samples whether they have multiple scattering, multiple modes of scattering, i.e. magnetic and lattice vibrations, or consist of a conglomeration of scatterers like a sample and sample environment. Additionally as the instrument is modeled component by component, instrument resolution is inherently included in the simulation.

As the promise of Monte Carlo ray tracing was clear, it has been used in codes that handle a subset of these complexities. MCS[10] and MSCAT[11] were early packages that used Monte Carlo methods to compute multiple scattering. 
Later, several general-purpose Monte Carlo neutron instrument simulation packages were developed and optimized to help design neutron instruments, namely McStas [12, 13], VITESS [14], IDEAS [15], and NISP [16]. Less-general-purpose MC programs exist in some popular neutron data analysis software packages, including DISCUS $[17,18,19]$ and RESTRAX $[20,21]$. In comparison, relatively few studies [22, 23, 24, 25, 26] have employed full-fledged Monte Carlo ray tracing to help analyze experimental results from neutron scattering measurements, while there are growing efforts to perform virtual neutron experiments using MC ray tracing [27, 28, 29, 30, 31]. MCViNE[32], an open source software, is designed to easily allow complex studies (see [9] for an example) and therefore should accelerate the use of Monte Carlo ray tracing in the analysis of experimental data.

The improvements in computing over the last decades include hardware developments, the emergence of object-oriented languages $\mathrm{C}++$ and Python, and advances in software design [33]. The amazing increases in hardware capabilities now allows detailed simulations to run in a reasonable time. The impact of modern software engineering practice on MC simulation codes is still progressing. MCViNE was developed, as part of the DANSE project [34], using such soft40 ware practices. Therefore it uses object oriented programing (OOP) concepts to represent instrument and sample components, following the hierarchical and modular nature of the neutron instruments, detector systems, and sample environments. This use of hierarchies from inside an instrument component or sample kernel up through the total instrument goes beyond the imperative pro${ }_{45}$ gramming paradigm, which is popular in other codes. ${ }^{1}$ By taking advantage of OOP design patterns as well as recursive algorithms, the MCViNE architecture supports an easily extensible library for scattering kernels suitable for both samples and detector systems. This approach allows for maximum flexibility, extensibility, and reuse of scatterer arrangements, geometrical shapes, and scattering mechanisms, and hence improves the sustainability of the software.

\footnotetext{
${ }^{1} \mathrm{~A}$ brief discussion is available in the supplemental material.
} 
This paper contains a description of the MCViNE software framework. Section 2 presents the challenges in simulating experiments carried out in modern neutron instrumentation, and an overview of the software engineering design and main software components and algorithms. Examples of MCViNE simulations for powder and single-crystal experiments are presented in Section 3. Concluding remarks follow in Section 4.

\section{Simulation of Spectra Measured at Modern Neutron Spectrome- ters with MCViNE}

\subsection{Challenges for simulating neutron scattering experiments}

Monte Carlo (MC) ray tracing simulations of neutron scattering spectrometers with support of multiple scattering were performed from 1970s using MCS[10] and MSCAT[11]. They were used to understand the effects of multiple scattering on the measured spectra [35]. In the 1990s, with increasing needs of simulating neutron instruments for the purpose of instrument design and optimization, several MC neutron ray tracing packages emerged, including McStas[12, 13], Vitess[14], Ideas[15], and NISP [16]. Simulations performed with these packages provide not only independent checks for analytical calculations of instrument performance, but also can be used to obtain optimal parameters for instrument design (see, for example, [36, 37, 38, 39, 40, 41, 42, 43, 44, 45]). Unlike MCS and MSCAT, most of these newer MC software packages (with a notable exception in NISP) treat simulation of a neutron instrument as a linear chain of neutron optical components, each of which modify some set of neutron beam characteristics such as spatial divergence and energy distribution. This linear approach greatly improved the computational efficiency and simplified the coding of instrument simulations. Such an approach is adequate because the physical formation of a neutron scattering instrument is linear, and the underlying hypothesis that neutrons at the downstream components are rarely scattered back to the upstream components holds up well in most instrument 
configurations. As a result, these software packages, especially McStas and Vitess, are making significant impacts for neutron instrument design.

For Monte Carlo neutron ray tracing simulations to be useful for interpretation of neutron scattering spectra, it is necessary to include detector systems and samples/sample environments, for which the physical arrangements are often non-linear. The detector systems in modern neutron scattering instrumentation display modularity, repetition, and sometimes hierarchical organization, reducing the engineering difficulties in manufacturing, testing, and validation. For example, the four direct geometry time-of-flight spectrometers at Spallation Neutron Source (SNS) [46], ARCS [47], SEQUOIA [48, 49], CNCS[50], and HYSPEC[51] share some instrument elements[52]: the Fermi chopper slit pack90 ages for the ARCS and SEQUOIA instruments are interchangeable, and the detector systems of all four instruments use the same so-called 8-packs [53], each of which is a detector pack consisting of eight ${ }^{3} \mathrm{He}$ linear position sensitive detector tubes (LPSD). The 8-packs are arranged in a vertically-oriented cylindrical geometry around the sample position, forming a hierarchical organization of pixels, tubes, packs, and detector rows. A simplified illustration of the detector hierarchy can be found in Figure 1(c).

Samples, sample holders, and sample environments are constituents of a "sample assembly", the term we use to refer to the collection of neutron scatterers near the sample position. A sample-assembly example is illustrated in ${ }_{100}$ Figure 1(b). A linear representation faces challenges in simulations of a sample assembly because it does not match physical arrangements of a sample or samples with associated sample environment components, and because neutrons can often scatter back and forth among the constituents. A good example of complex sample environments is the MICAS furnace [54]: the incident neutron beam intersects heating elements and up to 8 heat shields, all thin vertical hollow cylinders, in addition to the sample. The sample itself can be challenging too. For example, "single crystal" superconductor samples for inelastic neutron scattering experiments often consist of co-aligned arrays of small crystals mounted on an aluminum plate [55]. Another major difficulty stems from the 

composite kernel and scattering kernels. Currently two such components exist, 


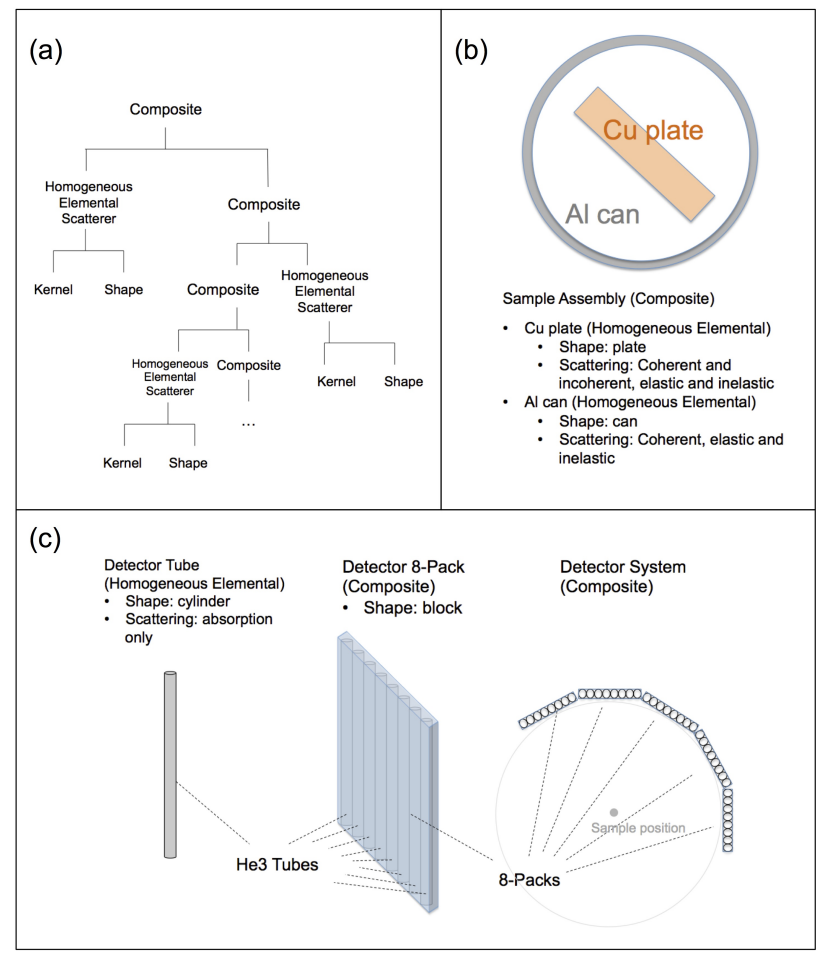

Figure 1: Concepts in scattering composites: composite scatterer, homogeneous scatterer, shape, scattering kernels. (a) an abstract hierarchy of an abitrary scattering composite. (b) and (c) are concrete examples of such hierarchies. (b) top view of a sample assembly consisting of an aluminum can and a copper plate. (c) a detector system - how it is constructed from an elemental scatterer (detector tube) in a three-level hierarchy.

one for sample assemblies and one for detector systems. This unique feature of MCViNE, the "composite scatterer", will be explained in detail in section 2.4.

A Python layer on top of the two $\mathrm{C}++$ layers allows construction of a component chain similar to McStas-like neutron ray tracing packages, provides the interface to the $\mathrm{C}++$ components to include in that chain, and allows for introduction of components that are completely written in Python. This last feature makes it extremely easy to create simple neutron components, and to create prototypes of more sophisticated neutron components. 


\subsection{Composite Scatterer}

This section starts with basic concepts with regard to the "composite scatterer", followed by an introduction to the essential object-oriented software designs centered around it, and finishes with examples of ray-tracing algorithms in sample assemblies and detector systems, enabled by these designs and needed in simulation of neutron scattering experiments in modern spectrometers.

\subsubsection{Concepts}

In MCViNE, a "composite neutron scatterer" represents a group of physical objects, for example, a powder sample in an aluminum can, a single crystal sample surrounded by a furnace, or a detector system. An "elemental scatterer" is a scatterer without constituent scatterers. A "homogeneous scatterer" is one kind of elemental neutron scatterer, whose scattering function is homogeneous within its volume. The scattering properties are modeled using one "scattering kernel" or a combination of several "scattering kernels", each of which represents one scattering mechanism, such as incoherent one-phonon nuclear scattering or coherent magnetic scattering.

These concepts are illustrated in Figure 1. Figure 1(a) is an example of an abstract hierarchy of a composite scatterer in MCViNE. In principle, the hierarchy in MCViNE can be arbitrarily deep. In practice, the depth of the hierarchy is limited by factors such as computing resources available for the simulations, and compiler limitations. Figure 1(b) depicts a sample assembly that is a composite of two-level hierarchy, in which the bottom level consists of two homogeneous elemental scatterers: one aluminum can and one copper plate sample. Figure 1(c) represents a detector system consisting of ${ }^{3} \mathrm{He}$ eightpacks that form roughly a cylindrical arrangement around the sample position. The detector system is represented in MCViNE in a three-level hierarchy: at the bottom level is the ${ }^{3} \mathrm{He}$ detector tube; at the middle level, 8 such tubes construct an 8-pack; at the top-level, the detector system consists of a collection of 8-packs. Such hierarchical representations allow MCViNE to model the physical reality closely. 


\subsubsection{Object Oriented Designs}

These concepts lead to one major design decision made in the MCViNE project: employment of the "composite design pattern", which is used to describe the part-whole relationship and to represent a hierarchy, and allows clients to treat composites and their constituents in a uniform way, and the "visitor design pattern", which allows separation of operations from the objects to be operated on, so that new operations can be added without touching these objects [33]. By using these design patterns for scattering composites, we can unify the programming interfaces to the operations on both the composites and the individual elemental objects. Composite and visitor patterns are used in three major aspects of the MCViNE neutron scattering model: the neutron scatterers, the geometric shapes of scatterers, and the scattering kernels.

Neutron scatterers. By using the composite pattern, algorithms for multiple scattering can be consolidated in one implementation. Scattering from a composite neutron scatterer starts with a determination of which constituent intersects the incident neutron ray, and then delegates the scattering assessment to that particular constituent, which could be a composite itself that requires another delegation for scattering. The hierarchical representation of neutron scatterers and this recursive algorithm work for both samples and detector systems, and can improve computing efficiency and code maintenance.

Geometric shapes Using constructive solid geometry (CSG) (see for example [56]), composite shapes are constructed from basic shapes such as cylinders and blocks, and composites by using operations such as union, intersection and difference. Ray-tracing through shapes is therefore simplified as visitor methods of the primitive shapes and the binary shape operations.

Scattering kernels Composite scattering kernels make it easy for the Monte Carlo algorithm to sample a total $S(\mathbf{Q}, \omega)$ consisting of both slowly-varying regions and regions containing sharp features, such as diffraction and coherent phonon scattering, by allowing users to combine different kernels such as incoherent and coherent kernels in a kernel composite. In addition, users can 
organize scattering kernels into groups; this makes it easy to apply importance sampling (assign different weights to different kernels or kernel groups) in simulations.

A "scattering kernel" in MCViNE is conceptually different from sample components in linear MC neutron ray tracing packages. A scattering kernel in MCViNE is an abstraction of the scattering mechanisms such as diffraction, nuclear scattering by phonons, and magnetic scattering by spin waves. It does not include the sample geometry but only the scattering physics. A sample component in earlier packages, on the other hand, includes both the geometry and the physics in one programming unit. By separating the implementation of "scatterer", "shape", and "scattering kernel", a sample in MCViNE can consist of a combination of scattering kernels. Furthermore, the scattering kernel library in the MCViNE framework can be extended without affecting the logic of geometric ray tracing, which is implemented in "shape" and "scatterer". For example, for isotropic scattering, scattering kernels taking a histogram form of $S(Q, \omega)$ can be supported, as well as phonon scattering kernels taking phonon energies and polarizations as inputs (examples are given in Section 3.1 and 3.2). A scattering kernel conveniently taking the analytical form of a dispersion can be used (see [9] for an example) to improve convergence (effective when 225 combined with other scattering kernels), and to avoid unnecessary broadenings resulted from approximating $S(Q, \omega)$ using a histogram. Inelastic and elastic scattering kernels for single crystals can also be developed to simulate single crystal experiments.

\subsubsection{Algorithms}

In this section, the general ray tracing procedure is first described briefly, and then implementation of multiple scattering and ray tracing in detector systems are presented as algorithm examples that benefit from the conceptual analysis and the software design.

\section{Ray Tracing}

235 In components such as sample assemblies or detector systems, neutron scat- 


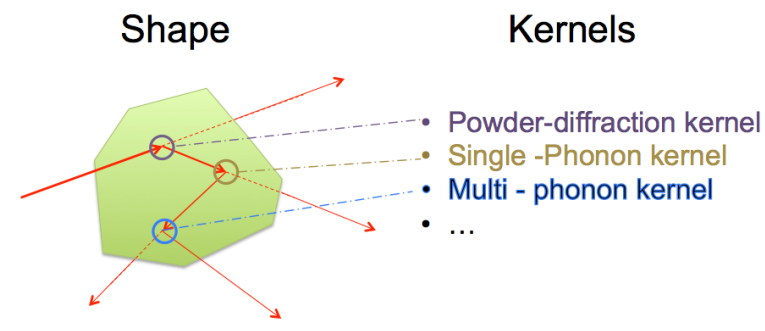

Figure 2: An example of multiple scattering within one scatterer. The incident neutron was scattered three times by three different scattering kernels. At each scattering point, the original neutron is also propagated out of the scatterer with proper attenuation. Red arrows are paths of neutron propagation. Circles highlight the location of scattering. Different scattering events are coded using different colors.

terers are represented by a hierarchy of objects with shapes and scattering mechanisms. Ray tracing of a neutron happens by first investigating which one of the neutron scatterers at the top level of the scatterer composite hierarchy intercepts the neutron. This is done by computing the intersections of the forward ray of the neutron and all the shapes of the top level constituents. A random selection might be necessary if multiple top-level objects intercept the neutron. After the top-level neutron scatterer is identified, the neutron is propagated to the front surface of the scatterer if necessary (not necessary if the neutron is already inside the scatterer) with appropriate attenuation, and then the ray tracing algorithm recurses into itself if the scatterer is a composite. Otherwise a point in the forward path of the neutron inside the scatterer will be randomly picked, and the neutron is propagated to that point with attenuation. At this point a scattering (or an absorption) mechanism of the scatterer is randomly picked, and the neutron will be either scattered with its probability adjusted, or be absorbed.

\section{Multiple Scattering}

Multiple-scattering (MS) is naturally supported in MCViNE scattering composites, implemented with a recursive algorithm. For the purpose of this discussion, we differentiate between two types of multiple scattering: single-scatterer 


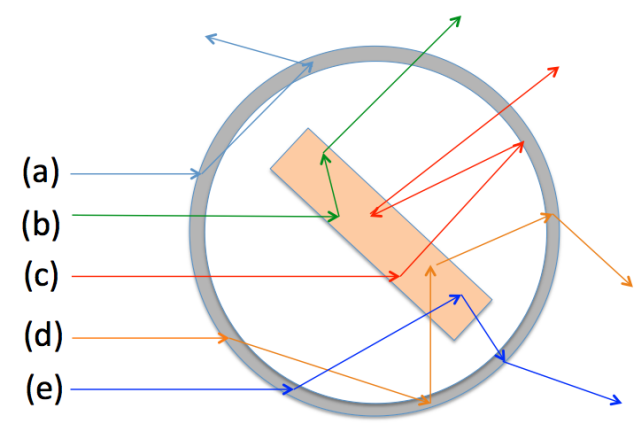

Figure 3: An example of multiple scattering in a concentric sample assembly. Five (out of infinite) possible multiple scattering paths are illustrated. Only five of an infinite number of possible multiple scattering paths are illustrated, and the splitting processes are not shown.

The multiple scattering algorithm of MCViNE is generic, and it can handle 
more complex sample assemblies, such as non-concentric arrangements, and mixing of non-concentric and concentric arrangements. More details about the MS algorithm of MCVINE can be found in the supplemental material for this paper.

Sample components in some linear-chain-based MC ray tracing packages can support SSMS, but they do not have abstractions similar to composite scatterer or scattering kernel. As a result, the SSMS algorithm must be duplicated in these sample components, while in MCViNE, implementations of new scattering kernels can be added without reimplementing the multiple scattering algorithm. McStas supports MSMS partially for a concentric sample assembly by, for example, adding a second outer cylinder into the simulation component chain [57]. Path (e) of Figure 3 is included this way, but path (c) of Figure 3 is not. Other ways of simulating MSMS may be possible with McStas, but these would require significantly more effort on the user's part.

The multiple scattering algorithm of MCViNE is more comparable to that of MSCAT[11] in which the sample and the sample environment are treated together in the multiple scattering loop, offering complete treatment of SSMS and MSMS. However, MCViNE allows a straightforward increase in complexity of the sample and sample environment interactions by using the recursive MS algorithm enabled by OOP. MSCAT by default only allows for a few specific arrangements. MSCAT and some McStas components contain the ability to appropriately transform the angular scattered distribution off of the sample so as to only simulate neutrons that will impact the detector, such optimization is not yet available in MCViNE.

\section{Ray tracing in a detector system}

Ray tracing of a neutron through a sophisticated detector system in instruments such as ARCS and SEQUOIA, where flat detector packs are arranged in an approximately cylindrical arrangement, illustrates another strength of the software design of MCViNE. MCViNE takes advantage of the hierarchical representation for neutron scatterers, only in this case the elemental homogeneous neutron scatterer is the ${ }^{3} \mathrm{He}$ detector tube that intercepts neutrons and records 
them. MCViNE reuses the code for ray tracing in a composite scatterer for simulating ${ }^{3} \mathrm{He}$ detector systems, and the new code needed is a scattering kernel for the ${ }^{3} \mathrm{He}$ material that takes into account gas absorption. The ray tracing through a cylinder takes care of the parallax effect of the detector tube. When a neutron is sent to a detector system shown in Figure 1(c), for example, the generic ray tracing algorithm for composite scatterers first checks whether the top level composite scatterer is penetrated by the neutron. If so, all constituents of the composite scatterer, i.e. the detector packs, are examined to determine which of them intercepts the neutron. Unless a neutron traverses a gap between detector packs, the detector pack is identified and then its constituents, the 8 detector tubes, are examined for neutron detection. The path of a neutron through the detector tube is then computed by ray tracing of the neutron through a cylinder, and a MC sampling picks a point in the path for the neutron to be absorbed. The position in the tube is used to calculate a unique pixel identifier known as the Pixel ID, according to the scheme readable by the Mantid software framework $[58,59]$. Additionally, the appropriate weighting multiplier for neutron probability (computed from absorption probability depending on the ${ }^{3} \mathrm{He}$ pressure and the length of the neutron path through the tube) and the time-of-flight are computed for the neutron to be recorded as a detector event in 325 a "virtual detector electronics device". Our hierarchical approach to detectors allows for the addition of more details, including details of the charge cloud and the wire if deemed necessary.

\section{Examples}

This section presents examples of MCViNE simulations performed for experiments on the ARCS, SEQUOIA, and HYSPEC instruments at SNS. These simulations were performed on one of the SNS data analysis clusters. A typical analysis cluster has 64 Intel or AMD CPU cores at $\sim 3 \mathrm{GHz}$, and simulations usually run in parallel using 10 cores. Every simulation consists of 4 steps:

Beam simulation. The incident beams on the sample for ARCS, SE- 

models were derived from the McStas[12] instrument definitions used in the design phase of the instruments[45, 48, 60, 61]. In this work, $10^{9}$ neutron events emitted from the moderator were included in all the simulations, and the neutrons at the sample position were saved and reused in the next step. A typical parallel run took about one hour.

Sample scattering. The neutron packets saved in the previous step were sent to a SampleAssembly component. Typically $10^{8}$ neutron packets were simulated in a run for any powder sample presented in this section, and it took minutes to hours to finish, depending on the complexity of the sample assembly. The scattered neutrons were then saved.

Detector interception. Each neutron scattered by the sample was processed by a DetectorSystem component and an event was recorded in an eventmode NeXus file if it intercepted a detector tube. In ray-tracing through a detector system, the detector tube in which the event was detected was located using a hierarchical set of detector tubes as described previously, while the exact location and time-of-flight were determined by a MC selection. The running time of this step depends on the number of neutrons hitting the detectors, which in turn depends on the number of neutron packets simulated in the previous step, as well as the user choice of multiple-scattering. Typically, $10^{8}$ 355 neutron packets took half an hour.

Reduction. The NeXus data generated in the previous step were reduced using Mantid[58, 59]. The only difference between the simulated and measured data is that the intensities in the simulations are computed as the sum of the probabilities of all packets arriving in the bin of interest, while those in the data are total event counts. The data reduction workflow is therefore identical, and uses the same code base for both the simulated and the measured data. This step typically took 10 s of minutes to finish for a powder measurement.

The goal of this four-step simulation process was to reproduce the experiment (including data reduction) by a simulation of high fidelity. 
lated scattered neutrons, the simulated event-mode NeXus file, and the reduced $I(Q, E)$ file.

The simulation examples here make use of the following scattering kernels that are described briefly in the Supplemental Material:

- incoherent elastic scattering

- coherent elastic scattering from powder sample

- incoherent inelastic single-phonon scattering

- coherent inelastic single-phonon scattering from a powder sample

- multi-phonon scattering

- scattering from a dispersion surface where the dispersion relation and the dynamical structure factor are described by analytical functions of momentum transfer vector $\mathbf{Q}$

Three examples are presented in the following subsections, two modeling vibrational excitations, and one modeling magnetic excitations. The simplest sample, a vanadium plate, is presented first in Subsection 3.1, where only incoherent scattering kernels are used. Coherent phonon scattering kernels for powder samples are introduced for an aluminum sample in Subsection 3.2. Both Subsection 3.1 and 3.2 demonstrate the ability to easily turn on and off different scattering mechanisms, allowing researchers to gain a better understanding of their contributions. Subsection 3.3 presents a simulation of a measurement on a single-crystal $\mathrm{K}_{2} \mathrm{~V}_{3} \mathrm{O}_{8}$ sample, for which a dispersion-surface scattering kernel is used.

In addition to these three examples, MCViNE simulations of Uranium Nitride (UN) measurements performed on the ARCS and SEQUOIA instruments provide an excellent example of the capabilities presented here [9]. In this case, the UN sample exhibited particularly strong multiple scattering due to its size. MCViNE simulations were able to reproduce well the multiple scattering, identified weak scattering from accoustic phonons, and showed that the binary solid 
model is a good explanation of the temperature-dependent broadening of the

\subsection{Vanadium}

Here we present experimental and simulated inelastic spectra for a $50 \mathrm{~mm} \times 50 \mathrm{~mm} \times 1.2 \mathrm{~mm}$ vanadium plate sample in the ARCS instrument. A quick calculation using the total scattering cross section for $\mathrm{V}$ shows that such a sample is a $6 \%$ scatterer. Vanadium, being the regular calibration standard in neutron scattering experiments, was chosen as the first example for its simplicity (incoherent scattering cross section is much larger than that of coherent scattering, and hence the scattering is isotropic). In the experiment, the sample was approximately perpendicular to the beam, and the incident energy was tuned to $117 \mathrm{meV}$ using a ing at $600 \mathrm{~Hz}$. In the simulation, the instrument parameters used matched the experimental ones. The simulation sample assembly contains only one homogeneous scatterer for the vanadium plate, which was tilted 96.6 degrees from the beam direction. Different scattering kernels were used for different simulations, but one incoherent elastic kernel and one single-phonon incoherent inelastic kernel were included for all. Incoherent scattering cross section of 5.08 barns and absorption cross section of 5.08 barns [62] were used. All phonon-releated scattering kernels use the phonon density of states (DOS) calculated from a Born-von Karman (BvK) model [63], which used force constants originally reported in Ref. [64], and tabulated in the Landolt Bornstein series of material properties [65]. Only one universal scale factor was applied to the intensities of all simulated spectra to match the experimental data.

Shown in Figure 4 are $I(Q, \omega)$ plots. The top panel is the experimental result. Panel (b) was simulated without either multi-phonon scattering or multiplescattering. Panel (c) was simulated with multi-phonon scattering but multiplescattering was turned off. Comparing (b) and (c), we can see how multi-phonon scattering contributes to intensities at high $Q\left(>\sim 10 \AA^{-1}\right)$, especially noticeable at high energy transfer above the single-phonon cut-off energy of $\sim 35 \mathrm{meV}$. 

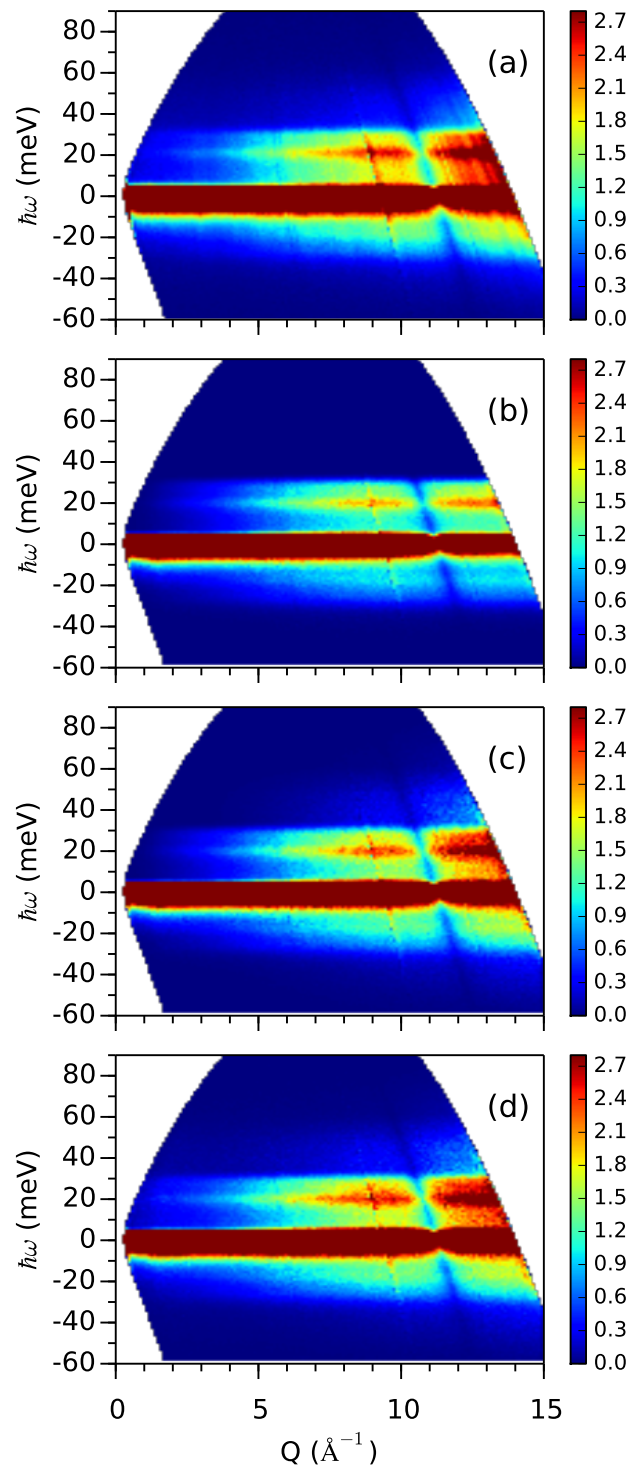

Figure 4: $I(Q, \omega)$ plots of vanadium inelastic spectra obtained from experiment and simulations for a vanadium plate at room temperature in the ARCS instrument. (a) Experiment (b) Simulation without a multi-phonon kernel. Multiple scattering was turned off. (c) Simulation with a multi-phonon kernel. Multiple scattering was turned off. (d) Simulation with a multi-phonon kernel. Multiple scattering was turned on. 


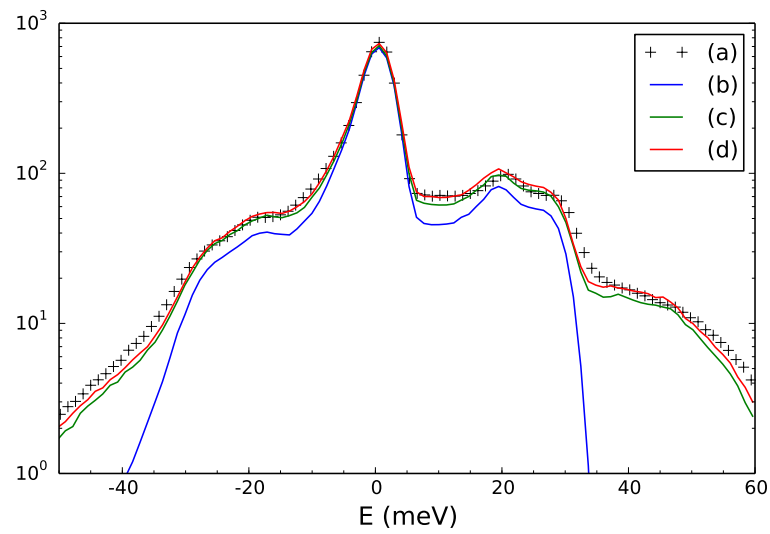

Figure 5: Energy spectra integrated over $\mathrm{Q}$ range $(8,12)$ inverse angstrom, obtained from experiment and simulations for a vanadium plate placed in the ARCS beam. The intensity axis is in log scale. (a)-(d) same as Figure 4

Panel (d) was done with both multi-phonon and multiple-scattering contributions. Comparing (c) and (d) shows how the effects of the multiple scattering are small but observable, particularly at low $Q$ values $\left(<\sim 2 \AA^{-1}\right)$. As the plate is only a $6 \%$ scatter, one would expect the multiple scattering to be less than $0.4 \%$, consistent with the observation. It also shows that there is less $Q$-dependence in multiple-scattering than in multi-phonon scattering. Figure 5 shows the energy spectra integrated over momentum transfer range of 8 to $12 \AA^{-1}$ in log scale. Similarly, we found that multi-phonon scattering contributes more substantially than multiple scattering in this $Q$ range.

To examine the agreement between the simulated and the experimental spectra, constant momentum cuts and constant energy cuts of the experimental data ${ }_{435}$ (Figure 4(a)) and the simulated data with the most complete collection of scattering kernels (Figure 4(d)) are presented in Figure 6 and Figure 7, respectively. The simulated data in general agree very well with the experimental data, across the measured dynamic range and the intensity range, showing features such as the asymmetric line shape of the elastic peaks, the detailed balance, and the Qdependent phonon-induced energy-loss profiles in the constant-momentum cuts, 

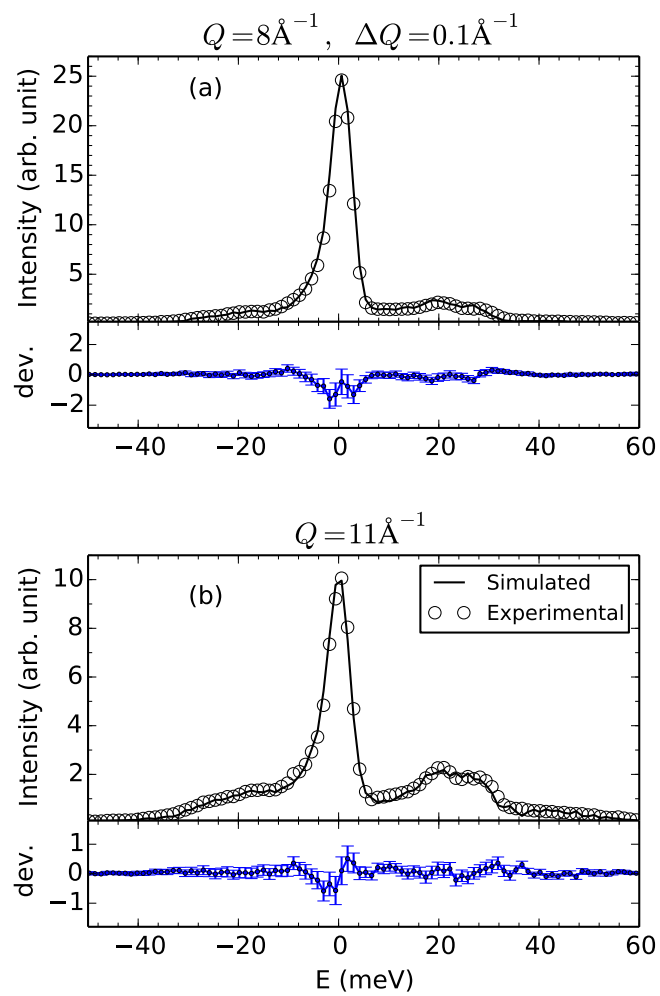

Figure 6: Constant momentum cuts of spectra as shown in Figure 4 at different $Q$ values: (a) 8 and (b) $11 \AA^{-1}$. The experimental cuts were taken from Figure 4(a), while the simulated cuts were taken from the best simulation incorporating all scattering kernels and multiple-scattering, Figure 4(d). The spectra show decreasing intensities in elastic peaks and increasing relative strength of inelastic scattering on both sides of the elastic peaks, with larger momentum transfers. The deviations of the simulated data from the experimental data ( $\Delta I=I_{\text {exp }}-I_{\text {sim }}$, similar thereafter) shown in the lower panels are small and are within error bars. The slightly larger deviations around elastic peaks may be attributed to slight mistmatch in determination of elastic lines, the fact that the coherent scattering is neglected in the simulations, as well as uncertainties in moderator profile, and in guide and chopper simulations. 

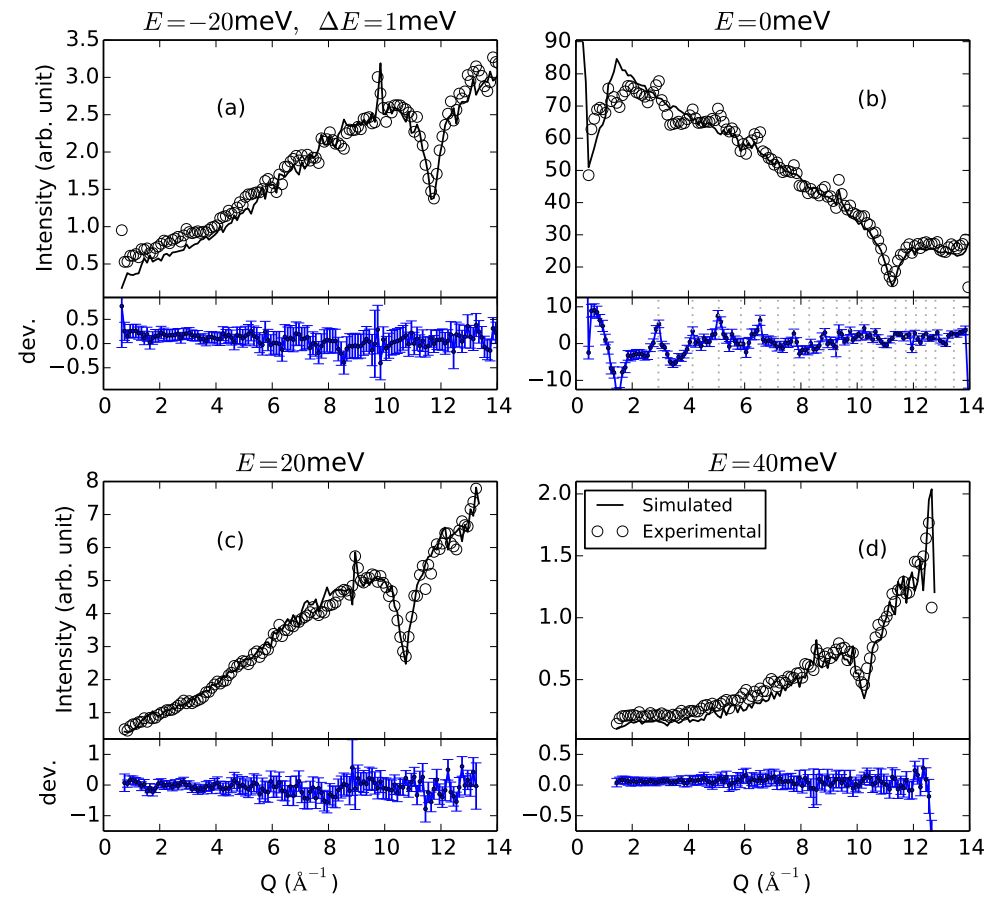

Figure 7: Constant energy cuts of spectra as shown in Figure 4 at different $E$ values: (a) -20 (b) 0 (c) 20 and (d) $40 \mathrm{meV}$. The experimental cuts were taken from Figure 4(a), while the simulated cuts were taken from the best simulation incorporating all scattering kernels and multiple-scattering, Figure 4(d). The elastic scattering in (b) weakens with higher $Q$ due to the Debye-Waller factor; the inelastic scattering in other panels strengthens with higher $Q$ : for panels (a) and (c), this trend is dominated by the one-phonon scattering proportional to the $Q^{2}$ factor in addition to the same Debye-Waller factor, while for panel (d) for which the energy transfer of $40 \mathrm{meV}$ is beyond the single-phonon energy cut-off, it is dominated by the two-phonon scattering proportional to the $Q^{4}$ factor. The dip shown in every panel is typical of the plate sample geometry and a result of absorption along the sample width (the dark angle). The deviations of the simulated data from the experimental one (shown in the lower panels) are in general smaller than error bars. The peaks in the deviation curve at the lower panel of (b) are beyond error bars, but they match positions of powder diffraction peaks as indicated by the vertical dotted lines, and most likely can be accounted for by the fact that the coherent scattering from $\mathrm{V}$ are neglected in the simulations. 
and the dark-angle dips in the constant-energy cuts. Also in the constant energy cuts (Figure 7), spikes in intensities (e.g., at $E=-20 \mathrm{meV}, Q \sim 10 \AA^{-1}$, and at $E=20 \mathrm{meV}, Q \sim 9 \AA^{-1}$ ) result from insufficient filling of the displayed $Q, \omega$ histogram bins by the detector pixels. While additional analysis may be able to correct for this effect, it is instructive that the detailed modeling of the ARCS detector array almost exactly matches the measured data, while typical MC simulations would miss this effect due to simplified detector models.

The uncertainties of this simulation arise from the following factors: deviation of the simulated incident beam from the actual neutron beam at the ARCS instrument; differences between the sample shape specification in simulation and the actual sample shape, such as its dimensions, and its orientation relative to the incident beam; the uncertainties in the physical and geometrical properties of the detector packs in the detector system, especially their positions and orientations; the deviation of the sample temperature in simulation from the actual temperature; and the uncertainties in the sample material such as impurities and contamination, neglect of Bragg reflections in simulations, and uncertainties in the phonon density of states (DOS) of vanadium.

The simulation of the incident beam is in part validated by comparison to the first beam monitor, and scattering from 2,5-diiodothiophene $\left(\mathrm{C}_{4} \mathrm{H}_{2} \mathrm{I}_{2} \mathrm{~S}\right)$ [47]. 460 This partial validation is corroborated by the agreement observed in the elastic peaks of $\mathrm{I}(E)$ spectra (Figure 6). The sample dimensions and its position and orientation influence the position of the elastic peaks, and the position and the width of the dark angle, which all show excellent agreement between experimental and simulated data. Uncertainties in positions and orientations of the detector packs can result in incorrect mapping from pixels to $Q, E$ coordinates. The fact that the simulated data show the same artifacts at the low-pixel-count detector area as the experimental data indicates that these errors are minimal. The deviation of experimental sample temperature from the simulation value is not expected to be larger than 10 Kelvin, which is consistent with excellent match in detailed balance between the simulation and the experiment shown in Figure 6. An examination of Figure 7(b) shows a series of sharp peaks, which 


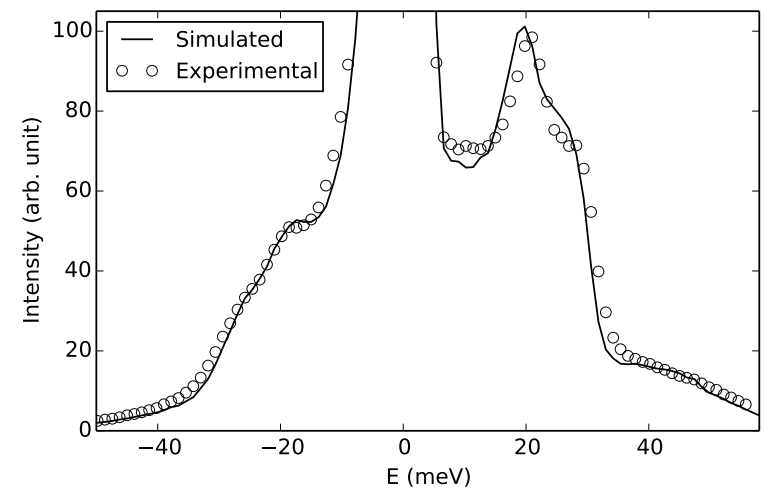

Figure 8: Experimental and best-simulated energy spectra obtained by integrating over Q range $(8,12)$ inverse angstrom of the spectra as shown in Figure 4 (a) and (d). The intensity scale is expanded to highlight the inelastic spectra.

arises from the Bragg reflections in the sample that were not included in the simulations. These may account for some differences in the elastic peaks in Figure 6 as well. The excellent agreement in all the other cuts of Figure 7 shows this does not affect the spectroscopic results. The remaining factor is the uncertainties in the phonon DOS, which is the main source of uncertainties in this simulation.

The discrepancy between the simulated data and the experimental data, relevant to researchers interested in phonons, is easier to observe from Figure 8. The double peaks near 20 and $28 \mathrm{meV}$ both appear to shift to the right in the experimental data, compared to the simulated one. This discrepancy has its origin in the phonon DOS used in the simulation, which is computed from a BvK model fitted to an X-ray determination of phonon dispersions[64]. Consistent with the discrepancies observed here, this BvK model slightly underestimated phonon energies when compared to a phonon DOS spectrum measured using inelastic neutron scattering (see Figure 10 of [64]). Actually, the uncertainty of measured vanadium phonon DOS reported over the years is larger than the discrepancy observed here (see, for example, Figure 2 of [66]). 


\subsection{Aluminum}

In this example, we present experimental and simulated inelastic spectra from a $60 \mathrm{~mm} \times 60 \mathrm{~mm} \times 4 \mathrm{~mm}$ polycrystalline aluminum (1100 alloy) plate in the ARCS instrument. A quick calculation using the total scattering cross section for $\mathrm{Al}$ shows that such a sample is a $5 \%$ scatterer. In the experiment, the sample was placed approximately at 135 degrees from the beam, and the incident energy was tuned to $80.5 \mathrm{meV}$ using the same Fermi chopper slit package as used in the previous example for vanadium, spinning at $480 \mathrm{~Hz}$. In the simulation, the instrument parameters used matched the experimental ones. The simulation sample assembly contains only one homogeneous scatterer for the aluminum plate. Different simulations made use of different combinations of scattering kernels. Tabulated Al cross sections [62] were used. All phonon-related scattering kernels use phonon energies and polarization vectors computed on a regular grid in a Brillouin zone from a BvK model [67]. The broadening of the phonon modes was not included. Only one universal scale factor was applied to all simulated spectra to match the experimental data.

Figure 9 shows $I(Q, \omega)$ plots for the aluminum plate. The experimental result is given in panel (a) and panels (b)-(e) show simulated data. In (b) only the incoherent elastic and incoherent single phonon scattering are included. In this plot the maximum intensity for the color scale is reduced by the ratio of incoherent/coherent cross sections of aluminum, so one can see the details in the plot. The numerical values on the colorbar give an indication of the scaling. In (c) only the coherent elastic (powder diffraction) and the coherent single-phonon inelastic scattering are included. In (d), all of the kernels in (b) and (c) with the addition of a multi-phonon kernel using the incoherent approximation. In (e), all of the kernels in (d) are used with multiple scattering turned on. Overall the features shown in the experimental data (a) and the simulated data (e) agree very well. Comparison of (b) and (c) shows that coherent scattering gives rise to more features such as diffraction peaks and phonon dispersion curves. It is evident from comparing (c) and (d) that multiphonon scattering increases in intensity at higher $Q$. The most obvious difference in (d) and (e) is in the elastic 

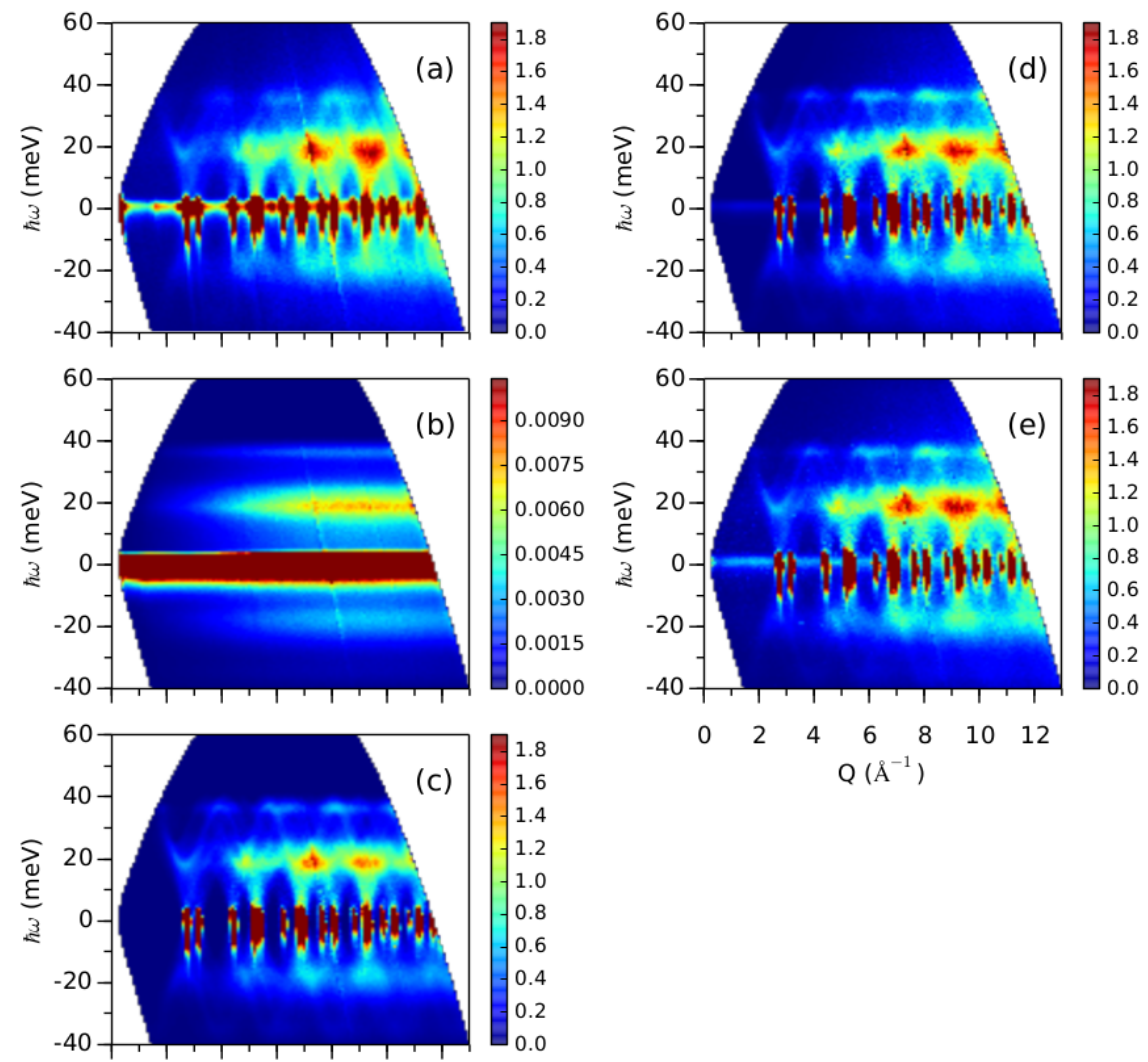

Figure 9: $I(Q, \omega)$ plots of aluminum inelastic spectra obtained from experiment (a) and simulations (b)-(e) of an aluminum plate at room temperature in the ARCS instrument. Scattering kernels included in the simulations are (b) incoherent elastic and incoherent inelastic single-phonon scattering (see note on intensity scaling in text), (c) coherent elastic (powder diffraction) and coherent inelastic single-phonon scattering, (d) all kernels included in (b) and (c) plus a multi-phonon kernel using an incoherent approximation and (e) all kernels included in (d) plus multiple scattering. 


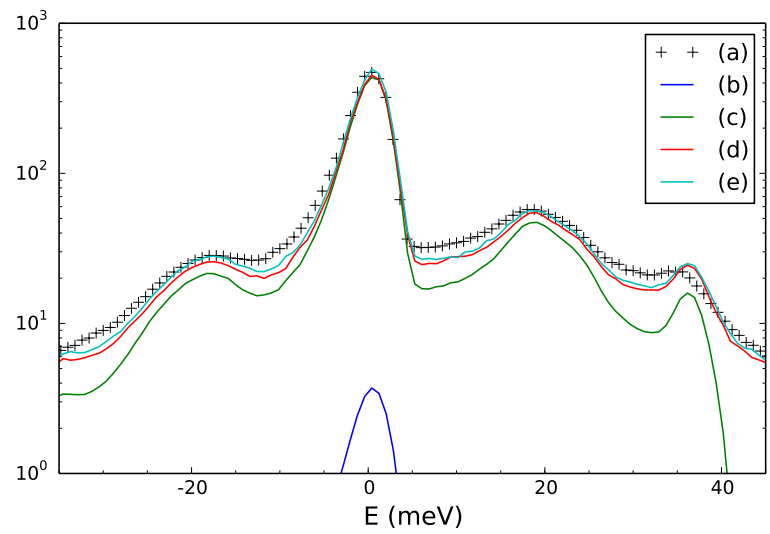

Figure 10: Energy spectra integrated over Q range $(6,10)$ inverse angstrom, obtained from experiment and simulations for an aluminum plate placed in the ARCS beam. The intensity axis is in log scale. (a)-(e) same as Figure 9

line, which shows that multiple scattering of coherent elastic scattering seems to contribute similarly to incoherent scattering in the elastic line. The elastic lines in (a) and (e) seem to show that the sample used in the experiment may contain traces of an additional phase, most likely from a surface layer of $\mathrm{Al}_{2} \mathrm{O}_{3}$. Figure 10 shows energy spectra integrated over a range of momentum transfer from 6 to $10 \AA^{-1}$ in $\log$ scale. Similar to the vanadium measurement, we found that multi-phonon scattering plays a significant role in the observed intensity, whereas multiple scattering plays little role in this $Q$ range. The is expected as the sample is only a $5 \%$ scatterer.

Similar to the vanadium study in the previous section, we further examine the agreement between the simulated and the experimental spectra by taking constant momentum cuts and constant energy cuts of the experimental data (Figure 9(a)) and the simulated data with the most complete collection of scattering kernels (Figure 9(e)). These cuts are presented in Figure 11 and Figure 12, and they again display strong agreement between the experimental and simulated data. For Figure 11, the maximum deviations occur near the elastic peak. Similarly, the biggest differences in Figure 12 are observed for the elas- 

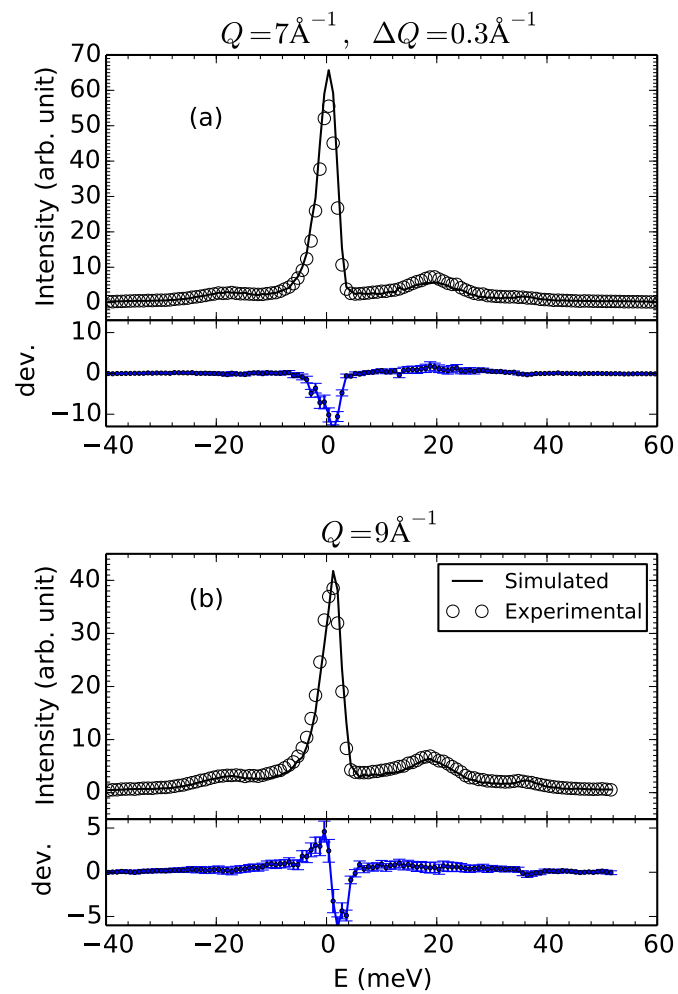

Figure 11: Constant momentum cuts of spectra as shown in Figure 9 at different $Q$ values: (a) 3 (b) 5 (c) 7 and (d) $9 \AA^{-1}$. The experimental cuts were taken from Figure 9(a), while the simulated cuts were taken from the best simulation incorporating all scattering kernels and multiple-scattering, Figure 9(e). The deviations of the simulated data from the experimental data are plotted in the lower panels. The main contribution to the larger deviations around the elastic peaks most likely comes from the texture in the sample. 

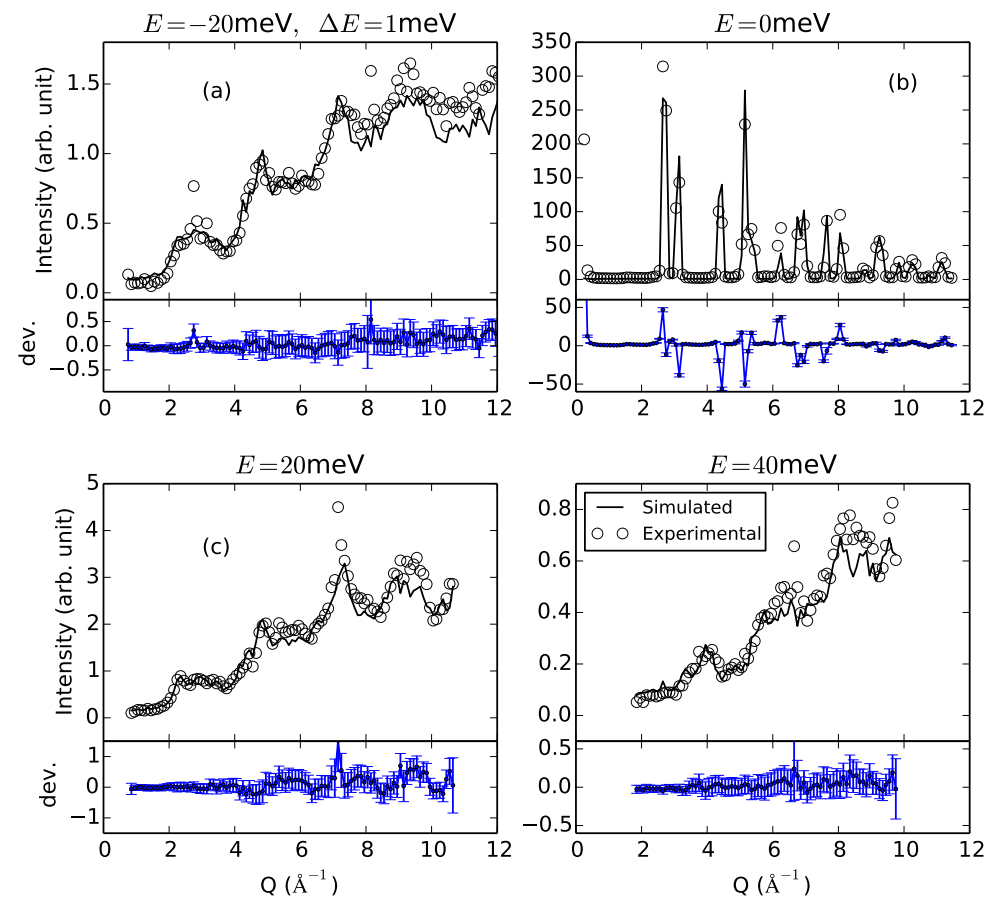

Figure 12: Constant energy cuts of spectra as shown in Figure 9 at different $E$ values: (a) -20 (b) 0 (c) 20 and (d) $40 \mathrm{meV}$. The experimental cuts were taken from Figure 9(a), while the simulated cuts were taken from the best simulation incorporating all scattering kernels and multiple-scattering, Figure 9(e). The deviations of the simulated data from the experimental one (shown in the lower panels) in general are smaller than the error bars. The discrepancies in (b) are from differences in the measured and calculated Bragg peak intensities. These arise from the fact that the sample is not a perfect powder. 
tic scattering in (b). These indicate that the measured Bragg peak intensities vary from the model: an isotropic powder average for Al. This could arise from texture, preferred grain orientation, or incomplete sampling of the powder[68].

The uncertainties of this simulation, like the case of vanadium, arise mainly from the uncertainties in the phonon data, which is the major input to all phonon-related kernels used in the simulation, only in this case the phonon data is not just a simple DOS curve. This phonon data input, existing in the form of energies and polarization vectors of all phonon branches, was computed for all points on a regular grid inside the first Brillouin zone, from a BvK model fit to phonon dispersions measured along the [100], [110], and [111] directions[67]. Small changes in the force constants in the BvK model can result in large fluctuations of the computed phonon energies and polarizations. Hence, the discrepancy between the experimental data (Figure 10(a)) and the simulated data (with all kernels, Figure 10(e)) was not unexpected. Another possible contributing factor is the fact that the broadening of $\mathrm{Al}$ phonon modes $[69,70]$ was not taken into account in our simulation.

\section{3. $\mathrm{K}_{2} \mathrm{~V}_{3} \mathrm{O}_{8}$ single crystal}

We now present experimental and simulated inelastic single-crystal spectra for a $\mathrm{K}_{2} \mathrm{~V}_{3} \mathrm{O}_{8}$ sample [71] measured at the HYSPEC instrument [51], a hybrid spectrometer at SNS with a focusing monochromator and a movable detector vessel. The sample consisted of 5 co-aligned crystals with an overall cylindrical shape approximately $3.8 \mathrm{~cm}$ in diameter and $2.5 \mathrm{~cm}$ in height. The sample was oriented so that at zero degrees of rotation angle its [100] direction was along the beam and its [001] direction pointed upward vertically. Measurements were performed at $1.5 \mathrm{~K}$ with an incident energy of $\sim 7 \mathrm{meV}$ with a Fermi chopper frequency of $180 \mathrm{~Hz}$. The detector vessel was oriented so that neutrons scattered from the sample in the horizontal plane were measured in the scattered angle range of -75 to -15 degrees. To span reciprocal space, the goniometer angle was swept from 40 to -50 degrees in 0.5 degree steps and then from -50 to -28 degrees in 1 degree steps. 
The simulation was done for a scan matching the experimental setup. The sample assembly contains one homogeneous scatterer for the $\mathrm{K}_{2} \mathrm{~V}_{3} \mathrm{O}_{8}$ sample with a shape matching the overall shape of the sample in the experiment. Two scattering kernels were included in the simulation: one incoherent elastic scattering kernel to approximate the elastic line, and one dispersion-surface scattering kernel for simulating the scattering from the spin-wave.

In $\mathrm{K}_{2} \mathrm{~V}_{3} \mathrm{O}_{8}$ the long-wavelength spin-wave dispersion is well described by a result for the quantum $(\mathrm{S}=1 / 2)$ square lattice Heisenberg antiferromagnet:

$$
E(\mathbf{Q})=2 \tilde{J} \sqrt{1-\gamma_{Q / /}^{2}}
$$

with the dynamic structure factor

$$
S(\mathbf{Q}, E)=\frac{1-\gamma_{Q_{/ /}}}{2 \sqrt{1-\gamma_{Q_{/ /}}^{2}}} \delta(E-E(\mathbf{Q}))
$$

where $\gamma_{Q_{/ /}}=\cos (h \pi) \cos (k \pi), 2 \tilde{J}=2.563$ [71]. The differential cross section is

$$
\left(\frac{d^{2} \sigma}{d \Omega d E_{f}}\right) \sim \frac{k_{f}}{k_{i}}|F(Q)|^{2}[n(E)+1]\left(1+\cos ^{2} \phi\right) S(\mathbf{Q}, E)
$$

where $k_{i}$ and $k_{f}$ are the magnitudes of the incident and final neutron wave vectors, $F(Q)$ is the magnetic form factor for $\mathrm{V}^{4+}[72], n(E)+1$ is the Bose occupation factor, and $1+\cos ^{2} \phi$ is a polarization term $(\phi$ is the angle between $\mathbf{Q}$ and the easy c-axis).

In the treatment of both experimental and simulated data, Mantid was used to reduce the measured NeXus file to NXSPE format, a specialized intermediate inelastic neutron data format, at each goniometer angle, and then a Python program was used to project data in NXSPE files to the four dimensional $\mathbf{Q}, E$ space. Slices along desired $\mathbf{Q}$ directions could then be taken.

Shown in Figure 13 are experimental and simulated slices along axes of $\mathbf{Q}=[h 10]$ and energy. The spin wave dispersion in the energy range between $\sim 0.3$ and $\sim 2.1 \mathrm{meV}$ shows good agreement between experimental and simulated data. In the experimental data, the noise in the energy range of $[0.25,0.7]$ is higher than the noise at higher energies. This is due to elastic scattering from 


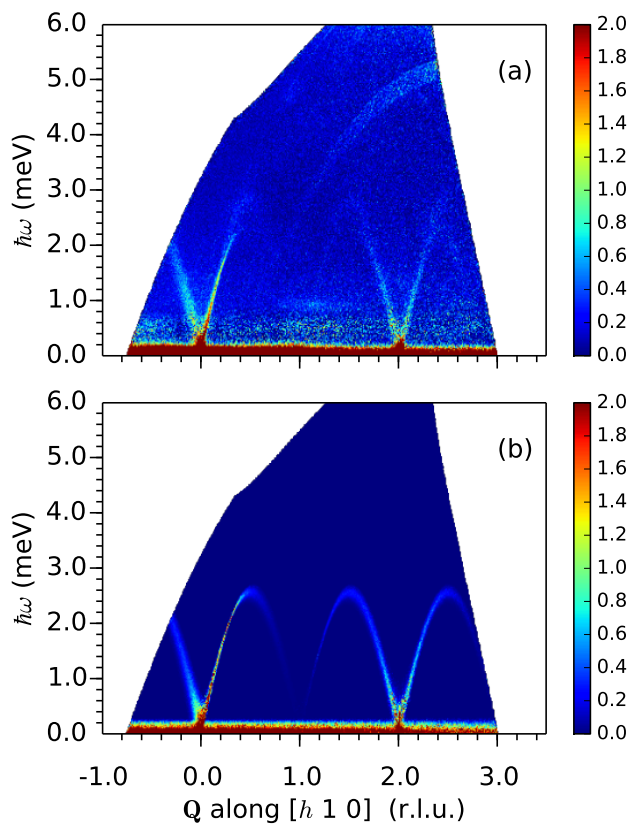

Figure 13: Slices along the $\mathbf{Q}=[h 10]$ axis and the energy axis from (a) experiment and (b) simulation, taken in the range $l=(-0.3,0.3)$ and $k=(0.93,1.07)$.

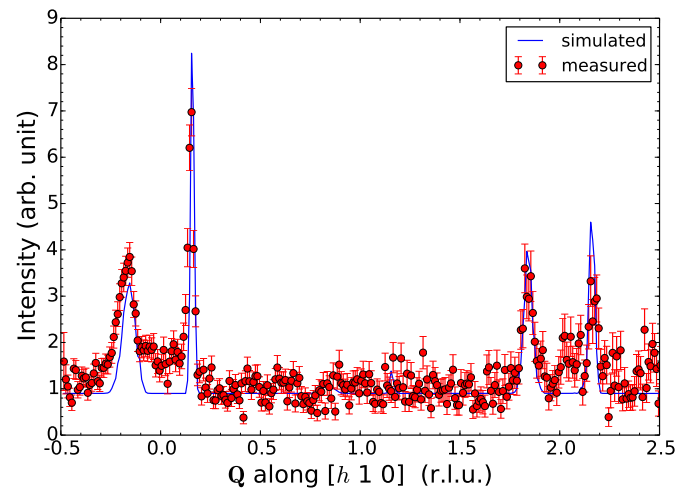

Figure 14: Constant energy cuts of the slices as shown in Figure 13 in the energy range of $[1.2,1.3] \mathrm{meV}$. A background term and a scaling factor were the only parameters used to fit the simulated data to experimental data. The goodness of fit, reduced $\chi^{2}$, was 2.56 . 
the cryostat, which was subtracted from the data. Both plots show a distinctive feature - for the dispersion near [010] the right branch is sharper than the left branch, while the dispersion branches near [210] are largely symmetric in their broadening. This effect is more clear in Figure 14, where constant-energy cuts over $[1.2,1.3] \mathrm{meV}$ are plotted against $\mathbf{Q}=[h 10]$ for the experimental (circles with error bars) and the simulated (solid line) intensities. Both the experimental data and simulated data show that the peak near $h=0.15$ is much sharper than that near $h=-0.15$. Such an effect is expected due to diffrent focusing conditions of the resolution ellipsoid [73, 74]. The spin-wave dispersion above $\sim 2.1 \mathrm{meV}$ ( $h \sim 0.33$, for example) has weaker intensity in the experimental data than expected from the simulated data, which could be attributed to mode splitting near the zone boundary [71].

\section{Conclusions}

The MCViNE software package for Monte Carlo neutron ray tracing simulations is based on modern object-oriented software design that decomposes neutron scatterers into a hierarchy of composite and elemental scatterers. This software design elegantly solves algorithmic problems including multiple scattering in a sample assembly, and ray tracing in a sophisticated detector system. Multiple scattering and instrument resolution are included naturally in MCViNE simulations, and simulation of ray-tracing in a complex detector system such as those of the ARCS and SEQUOIA instruments is straightforward. Adding, removing, and modifying scattering kernels in the simulations can help show the contributions of different types of scattering events to the experimental data. The examples of scattering from vibrational and magnetic excitations presented here and elsewhere[9] demonstrate that such simulations can improve our understanding of the underlying physics of neutron spectra.

Limitations of the MCViNE software package include (1) the complexity of sample assembly or detector system can be limited by available computing resources (memory and computation time); (2) it currently only supports ho- 
mogeneous scatterers, and this means materials with non-uniform distribution of defects, for example, can not be simulated without approximations; (3) it has a limited but expandable libary of scattering kernels. For example, for magnetic systems, it currently only supports spin-wave dispersions that can be expressed as analytical functions; (4) MCViNE currently relies on components adapted from other MC ray tracing packages to handle many neutron optical components such as guides and choppers, and thereby inherits their limitations.

MCViNE is freely available for the Linux platform. More details about the conditions of use and license can be found at http://danse.us/trac/MCViNE/wiki/license. Details on build and installation, usage, source repository, and user support of MCViNE are available in the documentation [32]. Feedback to the MCViNE developers can be provided through the MCViNE user mailing list http://groups.google.com/group/mcvineusers.

\section{Acknowledgements}

The development of the MCViNE software was begun by J.Y.Y.L. under the DANSE project supported by the NSF award DMR-0520547. The research on simulations of experiments in the ARCS, SEQUOIA, and HYSPEC instruments was supported by the U.S. Department of Energy, Office of Basic Energy Sciences. G.E.G., A.A.A., D.L.A., M.D.L., B.A. were fully supported, J.Y.Y.L. and H.L.S. partially supported by the Scientific User Facilities Division. We thank M. E. Hagen, A. Payzant, and P. Willendrup for stimulating discussions. We also thank L. Li and A. Dementsov for developing the powder diffraction scattering kernel for MCViNE, A. Fang for building MCViNE adaptations of some McStas components, and M. Reuter and S. Campbell for updating the MANTID code to read in the Monte Carlo generated data.

[1] V. Hugouvieux, E. Farhi, M. R. Johnson, F. Juranyi, P. Bourges, W. Kob, Structure and dynamics of l-ge: Neutron scattering experiments and ab initio molecular dynamics simulations, Phys. Rev. B 75 (2007) 104208. 
doi:10.1103/PhysRevB.75.104208.

URL http://link.aps.org/doi/10.1103/PhysRevB.75.104208

[2] A. C. Walters, T. G. Perring, J.-S. Caux, A. T. Savici, G. D. Gu, C.-C. Lee, W. Ku, I. A. Zaliznyak, Effect of covalent bonding on magnetism and the missing neutron intensity in copper oxide compounds, Nature Physics 5 (12) (2009) 867-872.

[3] O. Delaire, K. Marty, M. B. Stone, P. R. Kent, M. S. Lucas, D. L. Abernathy, D. Mandrus, B. C. Sales, Phonon softening and metallization of a narrow-gap semiconductor by thermal disorder, Proceedings of the National Academy of Sciences 108 (12) (2011) 4725-4730.

[4] F. Weber, S. Rosenkranz, L. Pintschovius, J.-P. Castellan, R. Osborn, W. Reichardt, R. Heid, K.-P. Bohnen, E. Goremychkin, A. Kreyssig, et al., Electron-phonon coupling in the conventional superconductor YNi2B2C at high phonon energies studied by time-of-flight neutron spectroscopy, Physical Review Letters 109 (5) (2012) 057001.

[5] A. A. Aczel, G. E. Granroth, G. J. MacDougall, W. J. L. Buyers, D. L. Abernathy, G. D. Samolyuk, G. M. Stocks, S. E. Nagler, Quantum oscillations of nitrogen atoms in uranium nitride, Nature Communications 3 (2012) 1124 .

[6] S. E. Hahn, A. A. Podlesnyak, G. Ehlers, G. E. Granroth, R. S. Fishman, A. I. Kolesnikov, E. Pomjakushina, K. Conder, Inelastic neutron scattering studies of $\mathrm{YFeO}_{3}$, Phys. Rev. B 89 (2014) 014420. doi : 10.1103/PhysRevB. 89.014420 .

URL http://link.aps.org/doi/10.1103/PhysRevB.89.014420

[7] K. W. Plumb, A. T. Savici, G. E. Granroth, F. C. Chou, Y.-J. Kim, Highenergy continuum of magnetic excitations in the two-dimensional quantum antiferromagnet $\mathrm{Sr}_{2} \mathrm{CuO}_{2} \mathrm{Cl}_{2}$, Phys. Rev. B 89 (2014) 180410. doi:10. 1103/PhysRevB.89.180410.

URL http://link.aps.org/doi/10.1103/PhysRevB.89.180410 
[8] C. W. Li, O. Hellman, J. Ma, A. F. May, H. B. Cao, X. Chen, A. D. Christianson, G. Ehlers, D. J. Singh, B. C. Sales, O. Delaire, Phonon self-energy and origin of anomalous neutron scattering spectra in snte and PbTe thermoelectrics, Phys. Rev. Lett. 112 (2014) 175501. doi: 10.1103/PhysRevLett.112.175501.

URL http://link.aps.org/doi/10.1103/PhysRevLett.112.175501

[9] J. Y. Y. Lin, A. A. Aczel, D. L. Abernathy, S. E. Nagler, W. J. L. Buyers, G. E. Granroth, Using Monte Carlo ray tracing simulations to model the quantum harmonic oscillator modes observed in uranium nitride, Phys. Rev. B 89 (2014) 144302. doi:10.1103/PhysRevB.89.144302.

URL http://link.aps.org/doi/10.1103/PhysRevB.89.144302

[10] F. G. Bischoff, Generalized Monte Carlo methods for multiple scattering problems in neutron and reactor physics, Ph.D. thesis, Rensselaer Polytechnic Institute (1970).

[11] J. R. D. Copley, P. Verkerk, A. A. Van Well, H. Fredrikze, Improved Monte Carlo calculation of multiple scattering effects in thermal neutron scattering experiments, Computer Physics Communications 40 (2) (1986) 337-357.

[12] K. Lefmann, K. Nielsen, McStas, a general software package for neutron ray-tracing simulations, Neutron News 10 (3) (1999) 20-23.

[13] P. Willendrup, E. Farhi, K. Lefmann, McStas 1.7 - a new version of the flexible Monte Carlo neutron scattering package, Physica B: Condensed Matter 350 (13, Supplement) (2004) E735 - E737, proceedings of the Third European Conference on Neutron Scattering. doi:http://dx.doi.org/10.1016/j.physb.2004.03.193. URL http://www.sciencedirect.com/science/article/pii/ S0921452604004144

[14] G. Zsigmond, K. Lieutenant, F. Mezei, Monte Carlo simulations of neutron scattering instruments by VITESS: Virtual instrumentation tool for ESS, Neutron News 13 (4) (2002) 11-14. arXiv: 
http://www.tandfonline.com/doi/pdf/10.1080/10448630208218488, doi:10.1080/10448630208218488.

URL http://www.tandfonline.com/doi/abs/10.1080/

10448630208218488

[15] W.-T. Lee, X.-L. Wang, J. Robertson, F. Klose, C. Rehm, IDEAS a Monte Carlo simulation package for neutron-scattering instrumentation, Applied Physics A 74 (1) (2002) s1502-s1504. doi:10.1007/s003390201723.

URL http://dx.doi.org/10.1007/s003390201723

[16] L. L. Daemen, P. A. Seeger, R. P. Hjelm, T. G. Thelliez, Monte Carlo tool for neutron optics and neutron scattering instrument design, SPIE Proc. 3771.

[17] T. Proffen, R. Neder, DISCUS: A program for diffuse scattering and defectstructure simulation, Journal of applied crystallography 30 (2) (1997) 171175.

[18] T. Proffen, R. Neder, DISCUS, a program for diffuse scattering and defect structure simulations-update, Journal of Applied Crystallography 32 (4) (1999) 838-839.

[19] T. Proffen, R. Neder, DISCUS.

URL http://discus.sourceforge.net/

[20] J. Šaroun, J. Kulda, Restrax - a program for tas resolution calculation and scan profile simulation, Physica B: Condensed Matter 234 (1997) 11021104.

[21] J. Šaroun, J. Kulda, RESTRAX.

URL http://neutron.ujf.cas.cz/restrax/

[22] V. Hugouvieux, E. Farhi, M. Johnson, W. Kob, Virtual neutron scattering experiments, Physica B: Condensed Matter 350 (13) (2004) 151 - 154, proceedings of the Third European Conference on Neutron Scattering. 
doi:http://dx.doi.org/10.1016/j.physb.2004.04.015.

URL http://www.sciencedirect.com/science/article/pii/

[23] L. Udby, P. K. Willendrup, E. Knudsen, C. Niedermayer, U. Filges, N. B. Christensen, E. Farhi, B. Wells, K. Lefmann, Analysing neutron scattering data using mcstas virtual experiments, Nuclear Instruments and Methods in Physics Research Section A: Accelerators, Spectrometers, Detectors and Associated Equipment 634 (1) (2011) S138-S143.

[24] P. L. Tregenna-Piggott, F. Juranyi, P. Christiansen, P. K. Willendrup, K. Lefmann, Reduction of data from inverted-geometry time-of-flight instruments, Journal of Neutron Research 16 (1-2) (2008) 13-22.

[25] M. Boin, A. Hilger, N. Kardjilov, S. Zhang, E. Oliver, J. James, C. Randau, R. Wimpory, Validation of bragg edge experiments by monte carlo simulations for quantitative texture analysis, Journal of Applied Crystallography 44 (5) (2011) 1040-1046.

[26] M. Boin, nxs: a program library for neutron cross section calculations, Journal of Applied Crystallography 45 (3) (2012) 603-607.

[27] K. Lefmann, P. K. Willendrup, L. Udby, B. Lebech, K. Mortensen, J. O. Birk, K. KlenØ, E. Knudsen, P. Christiansen, J. Saroun, et al., Virtual experiments: the ultimate aim of neutron ray-tracing simulations, Journal of Neutron Research 16 (3-4) (2008) 97-111.

[28] E. Farhi, V. Hugouvieux, M. R. Johnson, W. Kob, Virtual experiments: Combining realistic neutron scattering instrument and sample simulations, Journal of Computational Physics 228 (14) (2009) 5251-5261.

[29] Farhi, E., Willendrup, P., Virtual experiments in a nutshell: Simulating neutron scattering from materials within instruments with mcstas, Collection SFN 12 (2011) 303-339. doi:10.1051/sfn/201112015. URL http://dx.doi.org/10.1051/sfn/201112015 
[30] P. K. Willendrup, L. Udby, E. Knudsen, E. Farhi, K. Lefmann, Using mcstas for modelling complex optics, using simple building bricks, Nuclear Instruments and Methods in Physics Research Section A: Accelerators, Spectrometers, Detectors and Associated Equipment 634 (1) (2011) S150-S155.

[31] E. Farhi, C. Monzat, R. Arnerin, T. van Vuure, C. Castán-Guerrero, C. Hennane, P. Harraud, G. Campioni, S. Fuard, J. Ollivier, et al., Advanced sources and optical components for the mcstas neutron scattering instrument simulation package, Journal of Neutron Research 17 (1) (2014) $63-74$.

[32] J. Y. Y. Lin, M. A. Aivazis, B. Fultz, MCViNE.

URL http://docs.danse.us/MCViNE

[33] E. Gamma, R. Helm, R. Johnson, J. Vlissides, Design Patterns: Elements of Reusable Object-oriented Software, Addison-Wesley Longman Publishing Co., Inc., Boston, MA, USA, 1995.

[34] B. Fultz, et al., DANSE.

URL http://danse.us

[35] J. Wuttke, Multiple-scattering effects on smooth neutron-scattering spectra, Phys. Rev. E 62 (2000) 6531-6539. doi:10.1103/PhysRevE.62.6531. URL http://link.aps.org/doi/10.1103/PhysRevE.62.6531

[36] S. Kynde, K. H. Kleno, G. Nagy, K. Mortensen, K. Lefmann, J. Kohlbrecher, L. Arleth, A compact time-of-flight SANS instrument optimised for measurements of small sample volumes at the European Spallation Source, NUCLEAR INSTRUMENTS \& METHODS IN PHYSICS RESEARCH SECTION A-ACCELERATORS SPECTROMETERS DETECTORS AND ASSOCIATED EQUIPMENT 764 (2014) 133-141. doi: $\{10.1016 / j$.nima.2014.06.084\}.

[37] E. B. Klinkby, E. B. Knudsen, P. K. Willendrup, B. Lauritzen, E. Nonbol, P. Bentley, U. Filges, Application of the MCNPX-McStas interface 
for shielding calculations and guide design at ESS, in: Ioffe, A (Ed.), INTERNATIONAL WORKSHOP ON NEUTRON OPTICS AND DETECTORS (NOP\&D 2013), Vol. 528 of Journal of Physics Conference Series, Forschungszentrum Julich GmbH, Julich Ctr Neutron Sci; European Spallat Source Scandinavia; Swiss Neutron; Mirotron Ltd; S DH Heidelberg; ASTRIUM, 2014, International Workshop on Neutron Optics and Detectors (NOP\&D), Munich, GERMANY, JUL 02-05, 2013. doi : $\{10.1088 / 1742-6596 / 528 / 1 / 012032\}$.

[38] M. Bertelsen, H. Jacobsen, U. B. Hansen, H. H. Carlsen, K. Lefmann, Exploring performance of neutron guide systems using pinhole beam extraction, Nuclear Instruments and Methods in Physics Research Section A: Accelerators, Spectrometers, Detectors and Associated Equipment 729 (0) (2013) $387 \quad$ - 398. doi:http://dx.doi.org/10.1016/j.nima.2013.07.062.

URL http://www.sciencedirect.com/science/article/pii/ S0168900213010747

[39] O. Prokhnenko, K. Lieutenant, L. D. Cussen, W. D. Stein, C. Zendler, K. Prokes, Probing static and dynamic correlations in matter under extreme conditions: Concept of multi-purpose instrument at the European Spallation Source, NUCLEAR INSTRUMENTS \& METHODS IN PHYSICS RESEARCH SECTION A-ACCELERATORS SPECTROMETERS DETECTORS AND ASSOCIATED EQUIPMENT 764 (2014) 3041. doi: $\{10.1016 / j$.nima.2014.07.013\}.

[40] A. Houben, W. Schweika, T. Brckel, R. Dronskowski, New neutron-guide concepts and simulation results for the $\{$ POWTEX $\}$ instrument, Nuclear Instruments and Methods in Physics Research Section A: Accelerators, Spectrometers, Detectors and Associated Equipment 680 (0) (2012) 124 133. doi:http://dx.doi.org/10.1016/j.nima.2012.03.015.

URL http://www.sciencedirect.com/science/article/pii/ S0168900212002872 
[41] M. Skoulatos, K. Habicht, Upgrade of the primary spectrometer of the cold triple-axis spectrometer $\{$ FLEX $\}$ at the $\{$ BER $\}\{\mathrm{II}\}$ reactor, Nuclear Instruments and Methods in Physics Research Section A: Accelerators, Spectrometers, Detectors and Associated Equipment 647 (1) (2011) 100 106. doi:http://dx.doi.org/10.1016/j.nima.2011.05.037.

URL http://www.sciencedirect.com/science/article/pii/ S016890021100948X

[42] Z. Izaola, M. Russina, Virtual design of the neutron guide for the tof spectrometer neat, Journal of Physics: Conference Series 251 (1) (2010) 012064. URL http://stacks.iop.org/1742-6596/251/i=1/a=012064

[43] L. Alianelli, N. Wilson, K. Andersen, M. S. del Ro, R. Felici, A method for detailed simulations of neutron diffraction from imperfect crystals, Nuclear Instruments and Methods in Physics Research Section A: Accelerators, Spectrometers, Detectors and Associated Equipment 529 (13) (2004) 231 - 233, proceedings of the Joint Meeting of the International Conference on Neutron Optics (NOP2004) and the Third International Workshop on Position-Sensitive Neutron Detectors (PSND2004). doi:http://dx.doi.org/10.1016/j.nima.2004.04.161.

URL http://www.sciencedirect.com/science/article/pii/ S0168900204009039

[44] A. Wildes, J. Saroun, E. Farhi, I. Anderson, P. Hoghoj, A. Brochier, A comparison of monte-carlo simulation programs with experiment: the effect of a focusing guide on resolution, Applied Physics A 74 (1) (2002) s1452s1454. doi:10.1007/s003390101243.

URL http://dx.doi.org/10.1007/s003390101243

[45] G. E. Granroth, D. L. Abernathy, Performance comparisons of four direct geometry spectrometers planned for spallation neutron source performance comparisons of four direct geometry spectrometers planned for spallation neutron source performance comparisons of four direct geometry spectrom- 
eters planned for spallation neutron source, Proceedings of ICANS-XVI (2003) 289.

[46] T. Mason, T. Gabriel, R. Crawford, K. Herwig, F. Klose, J. Ankner, The spallation neutron source: A powerful tool for materials research, arXiv preprint physics/0007068.

[47] D. L. Abernathy, M. B. Stone, M. J. Loguillo, M. S. Lucas, O. Delaire, X. Tang, J. Y. Y. Lin, B. Fultz, Design and operation of the wide angularrange chopper spectrometer arcs at the spallation neutron source, Review of Scientific Instruments 83 (1) (2012) 015114-015114.

[48] G. E. Granroth, D. H. Vandergriff, S. E. Nagler, Sequoia: A fine resolution chopper spectrometer at the sns, Physica B: Condensed Matter 38586 (Part 2) (2006) 1104-1106.

[49] G. E. Granroth, A. I. Kolesnikov, T. E. Sherline, J. P. Clancy, K. A. Ross, J. P. C. Ruff, B. D. Gaulin, S. E. Nagler, Sequoia: A newly operating chopper spectrometer at the sns, Journal of Physics: Conference Series 251 (1) (2010) 012058.

[50] G. Ehlers, A. A. Podlesnyak, J. L. Niedziela, E. B. Iverson, P. E. Sokol, The new cold neutron chopper spectrometer at the spallation neutron source: Design and performance, Review of Scientific Instruments 82 (8) (2011) -. doi:http://dx.doi.org/10.1063/1.3626935.

URL http://scitation.aip.org/content/aip/journal/rsi/82/8/10. 1063/1.3626935

[51] B. Winn, U. Filges, V. Garlea, M. Graves-Brook, M. Hagen, C. Jiang, M. Kenzelmann, L. Passell, S. Shapiro, X. Tong, I. Zaliznyak, Recent progress on hyspec, and its polarization analysis capabilities, EPJ Web of Conferences.

[52] M. B. Stone, J. L. Niedziela, D. L. Abernathy, L. DeBeer-Schmitt, G. Ehlers, O. Garlea, G. E. Granroth, M. Graves-Brook, A. I. Kolesnikov, 
A. Podlesnyak, B. Winn, A comparison of four direct geometry time-offlight spectrometers at the spallation neutron source, Review of Scientific Instruments 85 (4) (2014)-. doi:http://dx.doi.org/10.1063/1. 4870050 .

URL http://scitation.aip.org/content/aip/journal/rsi/85/4/10. $1063 / 1.4870050$

[53] R. Riedel, R. Cooper, L. Funk, L. Clonts, Design and performance of vacuum capable detector electronics for linear position sensitive neutron detectors, Nuclear Instruments and Methods in Physics Research Section A: Accelerators, Spectrometers, Detectors and Associated Equipment 664 (1) (2012) 366 - 369. doi:http://dx.doi.org/10.1016/j.nima.2011.08.038.

URL http://ww.sciencedirect.com/science/article/pii/ S0168900211016597

[54] J. L. Niedziela, R. Mills, M. J. Loguillo, D. Armitage, H. L. Smith, J. Y. Y. Lin, M. S. Lucas, M. B. Stone, D. L. Abernathy, High temperature sample environments for inelastic neutron scattering at the spallation neutron source, in preparation.

[55] G. Friemel, M. Ohl, J. Park, B. Keimer, D. Inosov, Coaligning arrays of airsensitive single crystals for inelastic neutron scattering experiments, Journal of Physics: Conference Series 449 (1) (2013) 012016.

[56] S. Ghali, Introduction to Geometric Computing, 1st Edition, Springer Publishing Company, Incorporated, 2008.

[57] P. K. Willendrup, E. Knudsen, K. Lefmann, E. Farhi, Component Manual for the Neutron Ray-Tracing Package McStas.

URL http://www.mcstas.org/documentation/manual/

[58] O. Arnold, J.-C. Bilheux, J. Borreguero, A. Buts, S. I. Campbell, L. Chapon, M. Doucet, N. Draper, R. F. Leal, M. Gigg, et al., Mantiddata analysis and visualization package for neutron scattering and $\mu \mathrm{sr}$ 
experiments, Nuclear Instruments and Methods in Physics Research Section A: Accelerators, Spectrometers, Detectors and Associated Equipment 764 (2014) 156-166.

[59] J. Taylor, O. Arnold, J. Bilheaux, A. Buts, S. Campbell, M. Doucet, N. Draper, R. Fowler, M. Gigg, V. Lynch, et al., Mantid, a high performance framework for reduction and analysis of neutron scattering data, Bulletin of the American Physical Society 57.

[60] G. E. Granroth, M. Chen, J. A. Kohl, M. E. Hagen, J. W. Cobb, Fast Monte Carlo simulation of a dispersive sample on the sequoia spectrometer at the sns, Journal of Neutron Research 15 (1) (2007) 91-94.

[61] M. Hagen, B. Winn, V. Gosh, Private communication.

[62] V. F. Sears, Neutron scattering lengths and cross sections, Neutron News 3 (3) (1992) 26-37.

[63] A. A. Maradudin, E. W. Montroll, G. H. Weiss, I. P. Ipatova, Theory of lattice dynamics in the harmonic approximation, 2nd Edition, Academic Press, 1971.

[64] R. Colella, B. W. Batterman, X-ray determination of phonon dispersion in vanadium, Phys. Rev. B 1 (1970) 3913-3921. doi:10.1103/PhysRevB.1. 3913.

URL http://link.aps.org/doi/10.1103/PhysRevB.1.3913

[65] P. Dederichs, H. Schober, D. Sellmyer, Phonon States of Alloys. Electron States and Fermi Surfaces of Strained Elements, Vol. 13 of LandoltBornstein Group III Condensed Matter: Metals: Phonon States. Electron States and Fermi Surfaces, Springer-Verlag, Berlin, 1981.

[66] O. Gupta, M. Hemkar, Crystal equilibrium and lattice dynamics of vanadium, Il Nuovo Cimento B (1971-1996) 45 (2) (1978) 255-274. doi: 10.1007/BF02894684.

URL http://dx.doi.org/10.1007/BF02894684 
[67] G. Gilat, R. M. Nicklow, Normal vibrations in aluminum and derived thermodynamic properties, Phys. Rev. 143 (1966) 487-494. doi:10.1103/ PhysRev.143.487.

URL http://link.aps.org/doi/10.1103/PhysRev.143.487

[68] R. Young, The rietveld method, iucr monographs on crystallography 5, Oxford Science Publication.

[69] M. Kresch, M. Lucas, O. Delaire, J. Y. Y. Lin, B. Fultz, Phonons in aluminum at high temperatures studied by inelastic neutron scattering, Phys. Rev. B 77 (2008) 024301. doi:10.1103/PhysRevB.77.024301.

URL http://link.aps.org/doi/10.1103/PhysRevB.77.024301

[70] X. Tang, C. W. Li, B. Fultz, Anharmonicity-induced phonon broadening in aluminum at high temperatures, Physical Review B 82 (18) (2010) 184301.

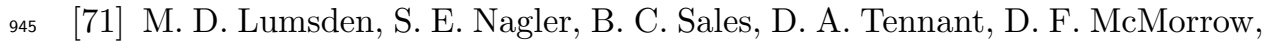
S.-H. Lee, S. Park, Magnetic excitation spectrum of the square lattice $\mathrm{S}=$ 12 heisenberg antiferromagnet $\mathrm{k}_{2} \mathrm{v}_{3} \mathrm{O}_{8}$, Phys. Rev. B 74 (2006) 214424. doi : 10.1103/PhysRevB.74.214424.

URL http://link.aps.org/doi/10.1103/PhysRevB.74.214424

950

[72] P. J. Brown, Magnetic form factors, Vol. C of International Tables for Crystallography, D. Reidel Publishing, Dordrecht, Holland, 1983-1993, Ch. 4.4.5, pp. 391-399.

[73] G. Shirane, S. M. Shapiro, J. M. Tranquada, Neutron Scattering with a Triple-Axis Spectrometer, Cambridge University Press, 2002, cambridge Books Online.

URL http://dx.doi.org/10.1017/CB09780511534881

[74] T. G. Perring, High energy magnetic excitations in hexagonal cobalt, dissertation, Cambridge University (1991). 